\title{
Granulocyte colony-stimulating factor alters the systemic metabolomic profile in healthy donors
}

\author{
Kimberley Joanne Hatfield $^{1,2}$ (D) Guro Kristin Melve $^{1,2} \cdot \emptyset_{\text {ystein Bruserud }}{ }^{1,3}$
}

Received: 7 July 2016/ Accepted: 4 November 2016/Published online: 28 November 2016

(c) The Author(s) 2016. This article is published with open access at Springerlink.com

\begin{abstract}
Introduction Peripheral blood stem cells mobilized by granulocyte colony-stimulating factor (G-CSF) from healthy donors are commonly used for allogeneic stem cell transplantation. The effect of G-CSF administration on global serum metabolite profiles has not been investigated before.

Objectives This study aims to examine the systemic metabolomic profiles prior to and following administration of G-CSF in healthy adults.

Methods Blood samples were collected from 15 healthy stem cell donors prior to and after administration of G-CSF $10 \mu \mathrm{g} / \mathrm{kg} /$ day for 4 days. Using a non-targeted metabolomics approach, metabolite levels in serum were determined using ultrahigh performance liquid chromatographytandem mass spectrometry and gas chromatography/mass spectrometry.

Results Comparison of the metabolite profiles of donors before and after G-CSF treatment revealed 239 metabolites that were significantly altered. The major changes of the metabolite profiles following G-CSF administration included alteration of several fatty acids, including increased
\end{abstract}

Electronic supplementary material The online version of this article (doi:10.1007/s11306-016-1139-x) contains supplementary material, which is available to authorized users.

Kimberley Joanne Hatfield

kimberley.hatfield@uib.no

1 Department of Clinical Science, University of Bergen, 5021 Bergen, Norway

2 Department of Immunology and Transfusion Medicine, Haukeland University Hospital, Bergen, Norway

3 Section for Hematology, Department of Medicine, Haukeland University Hospital, Bergen, Norway levels of several medium and long-chain fatty acids, as well as polyunsaturated fatty acids; while there were lower levels of other lipid metabolites such as phospholipids, lysolipids, sphingolipids. Furthermore, there were significantly lower levels of several amino acids and/or their metabolites, including several amino acids with known immunoregulatory functions (methionine, tryptophan, valine). Lastly, the levels of several nucleotides and nucleotide metabolites (guanosine, adenosine, inosine) were also decreased after G-CSF administration, while methylated products were increased. Some of these altered products/metabolites may potentially have angioregulatory effects whereas others may suggest altered intracellular epigenetic regulation.

Conclusion Our results show that G-CSF treatment alters biochemical serum profiles, in particular amino acid, lipid and nucleotide metabolism. Additional studies are needed to further evaluate the relevance of these changes in healthy donors.

Keywords Allogeneic stem cell transplantation .

Biochemical · Granulocyte colony-stimulating factor .

Metabolomics $\cdot$ Stem cell donor

\section{Introduction}

Peripheral blood stem cell (PBSC) grafts are commonly used for allogeneic hematopoietic stem cell transplantation for a wide range of hematologic malignancies. These grafts are generally prepared by administration of granulocyte colony-stimulating factor (G-CSF) to healthy donors which mobilizes hematopoietic stem cells (HSCs) into the blood before cells are harvested by leukapheresis. The clinical advantage of using PBSCs compared to bone marrow grafts 
includes accelerated neutrophil engraftment, which increases the likelihood to engraft, and in addition PBSC allografts contain a much larger number of T-cells compared to bone marrow grafts, which has been correlated with better outcome (Malard et al. 2016; Pabst et al. 2007; Rezvani et al. 2006).

The HSC mobilizing agent G-CSF is a glycoprotein with multiple functions, including effects on the production, migration, differentiation and proliferation of neutrophils, as well as affecting adaptive immune responses (Bendall and Bradstock 2014; Panopoulos and Watowich 2008). G-CSF may have both direct and indirect effects on immune cells, including monocytes, granulocytes, T-cells and dendritic cells, and can also alter the expression of various soluble factors, including cytokines, metalloproteinases and adhesion molecules which may themselves contribute to effects induced by G-CSF (Rutella et al. 2005). Recently, various metabolites and metabolic pathways have been found to be involved in cell signaling, also among immune cells; e.g. both amino acids as well as their metabolites can bind to specific receptors on immunocompetent cells and thereby induce activation and/or differentiation of these cells (Buck et al. 2015). Furthermore, certain metabolites have a key role in fundamental metabolic pathways, such as glycolysis or lipid metabolism, and their availability may thus affect immune cell functions. As reviewed by Buck et al. (2015), cellular metabolism is important in the regulation of immunocompetent cell growth, e.g. differentiation and activation of T-cells. Thus, the availability and uptake of metabolites may potentially affect immune cell fate. In this aspect, metabolomics has emerged as a powerful tool to identify and characterize the low molecular mass composition of biological samples. In this exploratory study, we therefore used non-targeted metabolomics to investigate the early effects of in vivo G-CSF treatment on the global serum metabolite profile of healthy stem cell donors.

\section{Materials and methods}

\subsection{Stem cell donors and mobilization}

The study was approved by the local ethics committee (REK Vest, 2011/996 and 2011/1237) and all samples were collected after written informed consent. Blood samples were collected from 15 consecutive healthy HLA-matched related allogeneic stem cell donors (10 males and 5 females), with a mean age of 47 years (range 25-64 years) (Table 1). Donors received the human non-glycosylated G-CSF analog Filgrastim (r-metHuG-CSF, Neupogen, Amgen) or Tevagrastim (biosimilar Filgrastim) in a dose of $5.4 \mu \mathrm{g} / \mathrm{kg}$ body weight (range $4.1-6.7 \mu \mathrm{g} / \mathrm{kg}$ ) twice daily subcutaneously for four days to induce stem cell mobilization. Our hospital is responsible for all allogeneic stem cell transplantations in a defined geographic area, and this study included a consecutive group of donors younger than 65 years of age and achieving pre-harvest $\mathrm{CD} 34^{+}$cell counts above $15 \times 10^{3} / \mathrm{L}$. Thus, our donors should be regarded as representative of healthy adult stem cell donors because they are unselected (i.e. consecutive), mobilize sufficient stem cells for preparation of allografts and their age is also representative for donors used in routine clinical practice.

\subsection{Processing of blood samples}

Venous blood samples were collected into Vacuette Z Serum Clot Activator tubes with Gel Separator (Greiner Bio-One GmbH, Kremsmünster, Austria) from donors at two time points, (i) prior to administration of G-CSF and (ii) following G-CSF administration just before apheresis on day 4. All samples were collected at 9 am and were allowed to coagulate for $30 \mathrm{~min}$ at room temperature in upright position before being centrifuged at $1310 \times g$ for $10 \mathrm{~min}$ at room temperature. The serum supernatants were immediately apportioned into $0.5 \mathrm{~mL}$ aliquots in plastic cryotubes (Nunc ${ }^{\mathrm{TM}}$, Roskilde, Denmark) and stored frozen at $-80^{\circ} \mathrm{C}$ until analysed.

\subsection{Analysis of G-CSF levels}

Levels of human G-CSF were measured using a Luminex assay (R\&D Systems, Bio-techne, Abingdon, UK), and the minimal detectable level was $20 \mathrm{pg} / \mathrm{mL}$.

\subsection{Analysis of human serum metabolites}

All mass spectrometry data were collected at Metabolon Inc (Durham, NC). Each serum sample was accessioned into the Metabolon LIMS system and was assigned a unique identifier by this system which was used to track all sample handling and results. All samples were prepared using the automated MicroLab STAR ${ }^{\circledR}$ system (Hamilton Company, Bonaduz, Switzerland). Briefly, samples were extracted using Metabolon's standard solvent extraction method (Evans et al. 2014). A recovery standard was added prior to the first step in the extraction process for quality control purposes. To remove protein, dissociate small molecules bound to protein or trapped in the precipitated protein matrix, and to recover chemically diverse metabolites, proteins were precipitated with methanol under vigorous shaking for 2 min followed by centrifugation. The resulting extract was divided into five fractions: (i) one for analysis by ultrahigh performance liquid chromatography-tandem mass spectrometry (UPLC-MS/MS) 
Table 1 Characteristics of allogeneic stem cell donors

\begin{tabular}{|c|c|c|c|c|c|c|c|c|}
\hline ID & $\begin{array}{l}\text { Age } \\
\text { (years) }\end{array}$ & Gender & $\begin{array}{l}\text { BMI } \\
\left(\mathrm{kg} / \mathrm{m}^{2}\right)\end{array}$ & $\begin{array}{l}\text { G-CSF } \\
\text { dosage } \\
(\mu \mathrm{g} / \mathrm{kg})\end{array}$ & $\begin{array}{l}\mathrm{G}-\mathrm{CSF}(\mathrm{pg} / \mathrm{mL}) \text { at } \\
\text { clinical examination }^{\mathrm{a}}\end{array}$ & $\begin{array}{l}\text { G-CSF }(\mathrm{pg} / \mathrm{mL}) \\
\text { before apheresis }^{\mathrm{a}}\end{array}$ & $\begin{array}{l}\mathrm{CD}_{3} 4^{+} \text {cell count } \\
\left(10^{3} / \mathrm{L}\right) \text { pre-harvest }^{\mathrm{b}}\end{array}$ & $\begin{array}{l}\mathrm{CD}^{2} 4^{+} \text {stem cell } \\
\text { yield }\left(\times 10^{6} / \mathrm{kg}\right)^{\mathrm{b}}\end{array}$ \\
\hline 1 & 60 & $\mathrm{~F}$ & 30 & 6.1 & $<20$ & $>15,000$ & 40.2 & 5.5 \\
\hline 2 & 25 & M & 24 & 6.1 & $<20$ & 13,514 & 44.4 & 8.8 \\
\hline 3 & 45 & M & 25 & 5.8 & 242 & $>15,000$ & 30.4 & 4.3 \\
\hline 4 & 51 & M & 47 & 4.9 & 37 & $>15,000$ & 58.8 & 3.9 \\
\hline 5 & 39 & M & 30 & 5.3 & 72 & 13,404 & 108.8 & 22.4 \\
\hline 6 & 64 & M & 36 & 4.1 & $<20$ & 9495 & 26.7 & 3.9 \\
\hline 7 & 54 & $\mathrm{~F}$ & 23 & 5.7 & 41 & $>15,000$ & 34.1 & 5.2 \\
\hline 8 & 25 & M & 26 & 5.7 & 111 & $>15,000$ & 147.8 & 15.1 \\
\hline 9 & 46 & F & 25 & 4.6 & 49 & 3939 & 57.6 & 7.2 \\
\hline 10 & 62 & M & 27 & 5.6 & 24 & $>15,000$ & 97.0 & 7.2 \\
\hline 11 & 51 & M & 26 & 5.1 & 53 & $>15,000$ & 17.4 & 3.1 \\
\hline 12 & 40 & $\mathrm{~F}$ & 34 & 5.3 & 39 & $>15,000$ & 66.7 & 6.8 \\
\hline 13 & 39 & M & 25 & 5.5 & 22 & $>15,000$ & 111.1 & 7.8 \\
\hline 14 & 45 & $\mathrm{~F}$ & 26 & 6.7 & 82 & $>15,000$ & 55.1 & 5.6 \\
\hline 15 & 58 & M & 29 & 5.1 & 80 & 6776 & 44.7 & 4.9 \\
\hline Mean & 47 & & 29 & 5.4 & 61 & $>13,000$ & 62.7 & 8.7 \\
\hline Range & $25-64$ & $\begin{array}{c}10 \mathrm{M} / \\
5 \mathrm{~F}\end{array}$ & $23-47$ & $4.1-6.7$ & $<20-242$ & $3939->15,000$ & $17.4-147.8$ & $3.1-22.4$ \\
\hline
\end{tabular}

$M$ male; $F$ female; $B M I$ body mass index

${ }^{\text {a }}$ G-CSF plasma levels were measured in donor samples collected at clinical examination and after four days of treatment with G-CSF before apheresis

${ }^{\mathrm{b}} \mathrm{CD} 34^{+}$cell counts were done immediately before stem cell harvest and the CD $34^{+}$stem cell yield estimated per $\mathrm{kg}$ donor/weight

with positive ion mode electrospray ionization, (ii) one for analysis by UPLC-MS/MS with negative ion mode electrospray ionization, (iii) one for LC polar platform, (iv) one for analysis by gas chromatography/mass spectrometry (GC-MS) and (v) one sample was reserved for backup. Samples were placed briefly on a Zymark TurboVap ${ }^{\circledR}$ (McKinley Scientific, Sparta, NJ, USA) to remove the organic solvent. Then samples were either stored overnight under nitrogen for $\mathrm{LC}$ or dried under vacuum overnight for GC, before preparation for analysis. Experimental samples were randomized across the platform and run with appropriate quality control samples spaced evenly among the injections. Compounds were identified by comparison to library entries based upon retention time/index, mass to charge ratio $(\mathrm{m} / \mathrm{z})$ and chromatographic data (also MS/MS spectral data), and peaks were quantified using area-underthe curve.

\subsection{Statistical analyses}

Two types of statistical analysis were performed: (1) significance tests (t-tests) and (2) classification analysis. Random Forest analysis is a supervised classification technique that provides an unbiased estimate of how well individuals can be classified into each group in a new data set. Statistical analyses were performed with the program $\mathrm{R}$ (http://cran.r-project.org/).

\section{Results}

\subsection{G-CSF treatment alters the global metabolomic profile of healthy individuals}

Metabolites were analysed in all serum samples collected from the healthy donors (i) prior to G-CSF administration and (ii) on day 4 after G-CSF administration. In total, 641 metabolites were identified (for a complete list see Supplementary Table 1), where levels of 239 metabolites were significantly changed $(\mathrm{p} \leq 0.05) ; 149$ metabolites had increased levels (62\%) and 90 metabolites had decreased levels (38\%) after G-CSF administration (Table 2). These significantly altered metabolites belong mainly to amino acid and lipid classes, while metabolites associated with the categories nucleotides, carbohydrates, energy metabolism, cofactors/vitamins and xenobiotics are also present. Furthermore, 39 of these metabolites have a p value $<0.0001$ and are involved in amino acid (19/39), 
Table 2 Metabolite classes significantly altered after G-CSF treatment

\begin{tabular}{|c|c|c|c|c|}
\hline \multirow[t]{2}{*}{ Metabolite classes/pathways } & \multirow{2}{*}{$\begin{array}{l}\text { Total number of } \\
\text { metabolites identified }\end{array}$} & \multicolumn{3}{|c|}{ Number of significantly altered metabolites } \\
\hline & & $\mathrm{P}<0.05$ & $\mathrm{P}<0.001$ & $\mathrm{P}<0.0001$ \\
\hline Amino acids & 161 & 75 & 30 & 19 \\
\hline Peptide & 28 & 16 & 1 & 1 \\
\hline Carbohydrates & 25 & 8 & 5 & 2 \\
\hline Energy metabolism & 9 & 2 & - & - \\
\hline Lipids & 250 & 78 & 13 & 5 \\
\hline Nucleotides & 34 & 21 & 11 & 10 \\
\hline Cofactors-vitamins & 22 & 10 & 4 & - \\
\hline Xenobiotics & 112 & 29 & 4 & 2 \\
\hline Total number of metabolites & 641 & 239 & 68 & 39 \\
\hline
\end{tabular}

nucleotide (10/39) or lipid (5/39) metabolism (Table 2). Among the significantly altered levels of metabolites ( $\mathrm{n}=239, \mathrm{p} \leq 0.05)$ we would expect to see approximately 12 metabolites that meet our level of significance criteria by random chance, however, our data have a low false discovery rate (FDR) of less than $5 \%$ for all metabolites except one metabolite with $5.1 \%$, indicating a high level of confidence in the results (Supplementary Table 2). All significantly altered metabolites $(\mathrm{p} \leq 0.05)$ and their group mean ratios (before vs. after G-CSF treatment) are shown in Supplementary Table 2.

We performed a principal component analysis (PCA) which showed that the samples before and after G-CSF administration were generally distinguishable from each other (Fig. 1); one G-CSF treated sample seemed to deviate from the other samples but no outlier samples were identified. This exceptional stem cell donor had the highest total leukocyte and platelet counts in peripheral blood, the lowest $\mathrm{Hb}$ level after G-CSF administration and the lowest G-CSF plasma level before apheresis, but did not otherwise differ from the others, and samples from this donor were included in all our analyses. Taken together, these results demonstrate that four days of G-CSF administration alters the systemic metabolomic profile of healthy individuals; and despite some heterogeneity between donors, an altered amino acid, lipid and nucleotide metabolism seems to be a common characteristic.

\subsection{Alteration of single metabolites by G-CSF treatment}

Random forest classification was used for further statistical analyses. Even though there was an overlap between samples collected before and during G-CSF therapy in the PCA plot (Fig. 1), the random forest classification demonstrated that G-CSF treated versus untreated samples

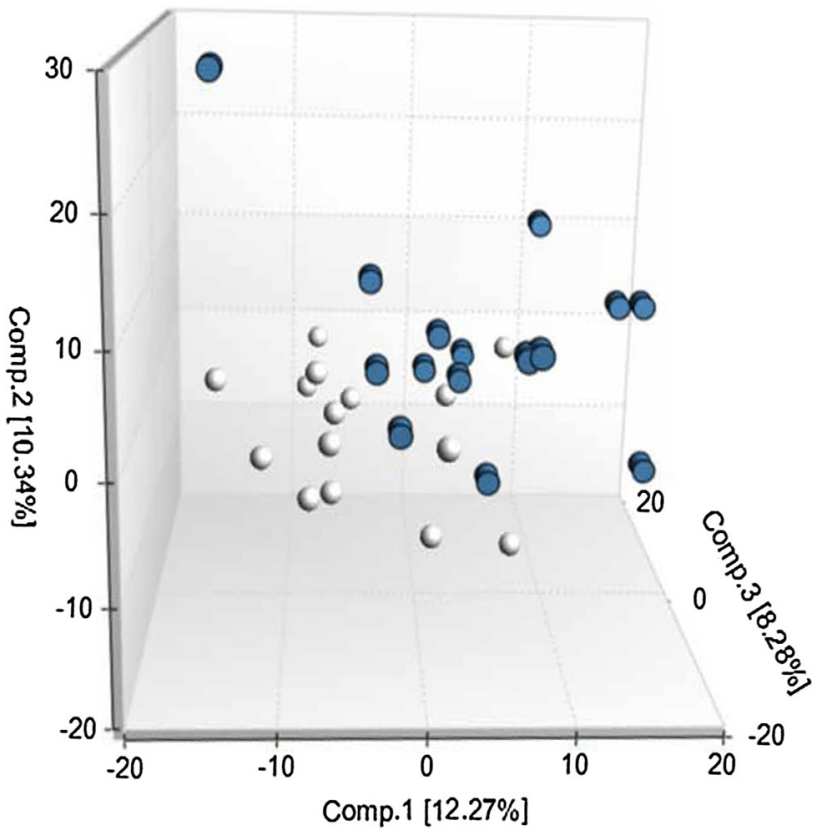

Fig. 1 Principal component analysis (PCA) scores plot based on the serum metabolome of healthy stem cell donors before and after G-CSF treatment. An overlap was seen between groups $(n=15$ in each group, open circles before G-CSF treatment, filled circles after G-CSF treatment), but groups were generally distinguishable from each other

could be distinguished with $97 \%$ predictive accuracy based on their overall metabolite profiles (Fig. 2). The 30 topranking metabolites that contributed most to separation of the samples are shown where the metabolites are ranked according to the mean decrease accuracy (\%) (Fig. 2). These metabolites are involved in several pathways, with the majority of metabolites belonging to amino acid metabolism ( 9 metabolites) and nucleotide metabolism (9 metabolites), but lipid (4 metabolites), xenobiotics (3 metabolites), carbohydrate (2 metabolites), cofactors/ 
Fig. 2 Random forest analysis of the metabolic profiles in samples taken before and after administration of G-CSF in healthy stem cell donors. Random forest analysis could distinguish between the metabolic profiles of the two groups with a predictive accuracy of $97 \%$. The variable importance plot shows the variable on the y-axis, and their importance for separation of the two groups on the $\mathrm{x}$-axis. The top-ranked 30 metabolites are thus ordered top-to-bottom as most- to least-important based on their importance. The inset table shows the main signaling pathways where each metabolite belongs, reflected by the different colors in the plot. Asterisk indicates that the biochemical name is identified but has not been confirmed based on a standard

\begin{tabular}{|l|c|c|c|}
\hline & \multicolumn{2}{|c|}{ Predictive } & \\
\hline Actual & Pre & Post & Class Error \\
\hline Pre & 14 & 1 & 0.067 \\
\hline Post & 0 & 15 & 0.000 \\
\hline \multicolumn{4}{|c|}{ Predictive accuracy $=97 \%$} \\
\hline
\end{tabular}

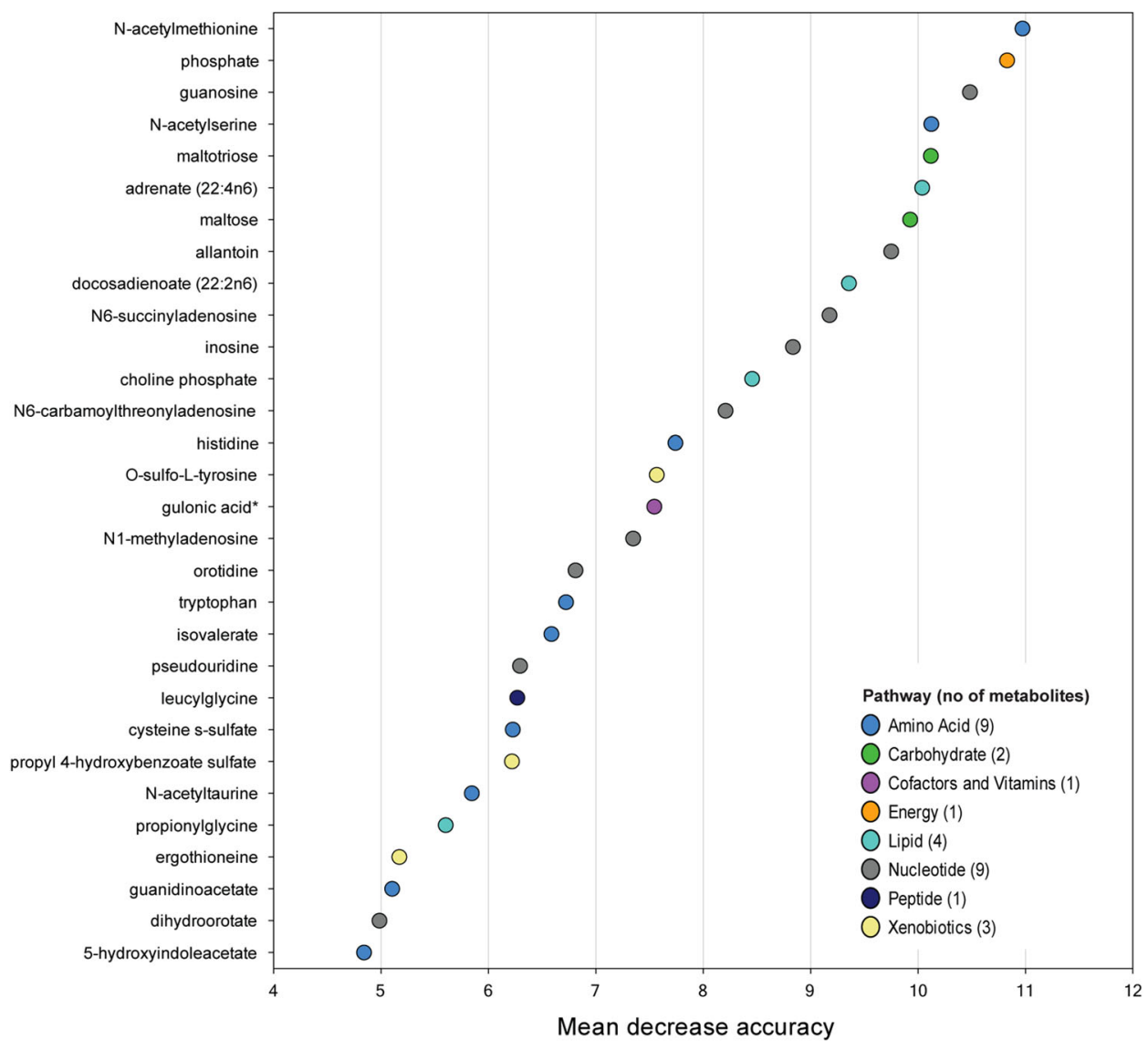

vitamin (1 metabolite) and peptides (1 metabolite) were also included among the 30 top-ranking metabolites in this analysis.

\subsection{Alteration of lipid, amino acid and nucleotide metabolism by G-CSF treatment}

Treatment with G-CSF resulted in altered systemic (i.e. serum) levels of a wide range of metabolites reflecting an alteration of different metabolic pathways during this treatment in healthy individuals:

- Lipid metabolism Among the main metabolites that distinguished between pre-and post-G-CSF administration groups, there was a consistent alteration of the amount of fatty acids indicating altered lipid metabolism (i.e. a change in fatty acid synthesis, lipid hydrolysis or mitochondrial $\beta$-oxidation) (Fig. 3a). The post-G-CSF group had significantly elevated levels of several long-chain fatty acids (such as myristate, palmitate, margarate, and stearate), as well as polyunsaturated fatty acids (adrenate, linoleate, linolenate, dihomolinoleate, docosadienoate, docosapentaenoate). Finally, levels of carnitine-conjugated lipids were increased.

- Amino acid metabolism was also altered; within the post-G-CSF samples there was a reduced level of dipeptides and amino acids including the aromatic amino acids tryptophan, phenylalanine and tyrosine and their metabolites, as well as branched-chain amino acids valine, isoleucine and leucine (Fig. 3b). The essential amino acid tryptophan can be metabolized by several pathways to give rise to serotonin or kynurenine, and levels of these degradation products as well as indoleacetate and 3-indoxyl sulfate were all significantly lower after G-CSF therapy.

- Nucleic acid metabolism Our results show a significantly lower level of purine nucleosides, including guanosine, adenosine and inosine in the post-G-CSF 
Fig. 3 Metabolite pathways altered after G-CSF administration among the classes lipids, amino acids and nucleotides. The bar charts show the different amounts of metabolites belonging to their different pathways within the three main classes: a lipid metabolism, $\mathbf{b}$ amino acid metabolism and $\mathbf{c}$ nucleotide metabolism, where metabolite levels were either increased (dark grey colored) or decreased (light grey)
A

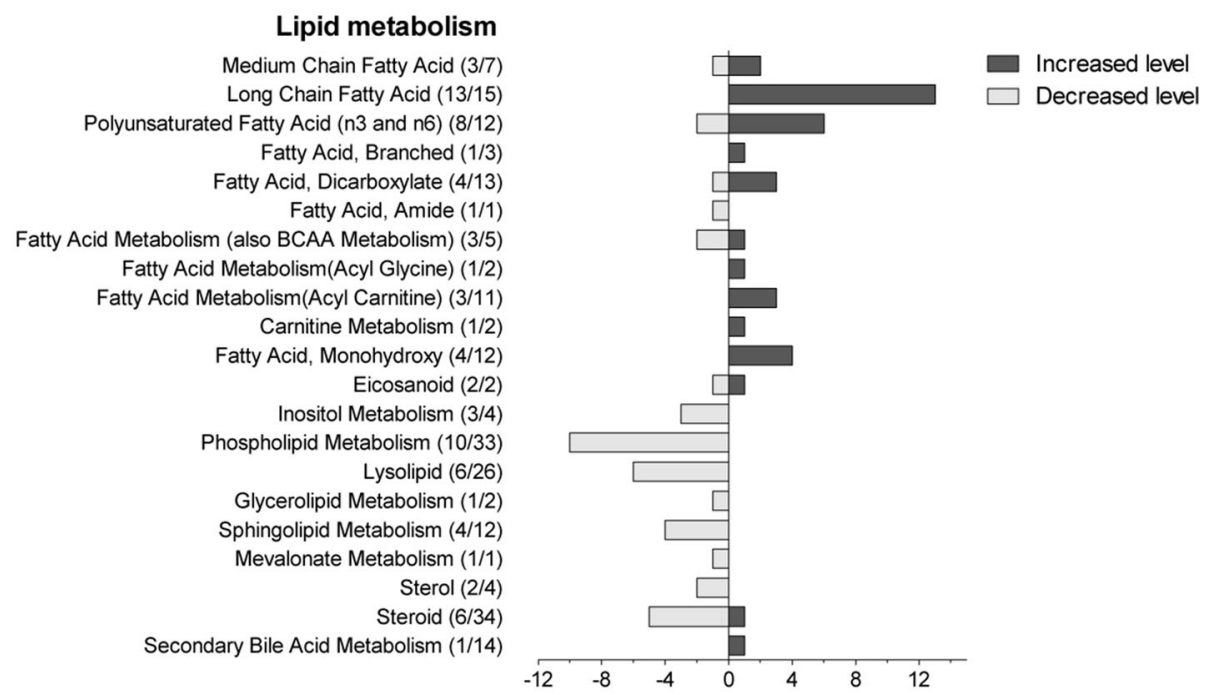

B

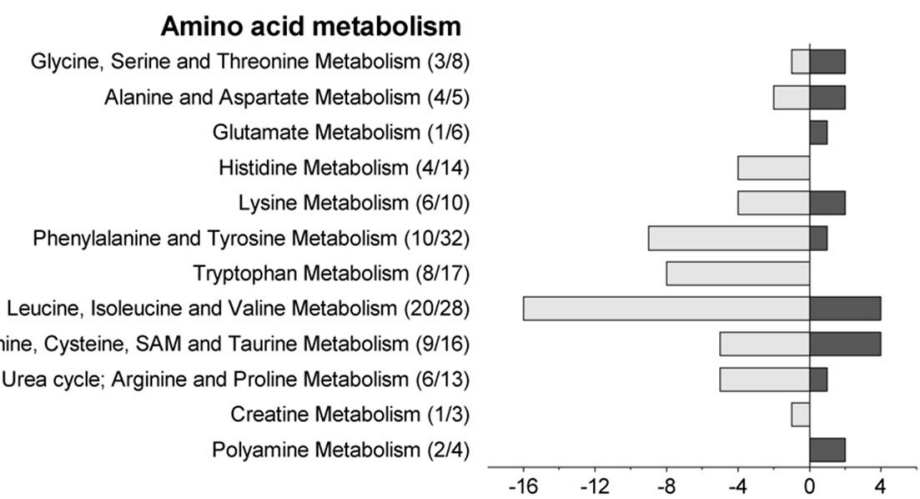

C

Nucleotide metabolism
Pyrimidine Metabolism, Cytidine containing (1/1)
Pyrimidine Metabolism, Uracil containing ( $7 / 10)$
Pyrimidine Metabolism, Orotate containing $(2 / 3)$
Purine Metabolism, Guanine containing (4/5)
Purine Metabolism, Adenine containing (5/7)

Purine Metabolism, (Hypo)Xanthine/Inosine containing (2/6)

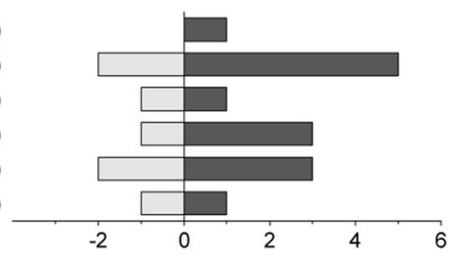

samples, indicating altered nucleotide metabolism (Fig. 3c). Furthermore, several methylated products were increased.

\subsection{G-CSF induced alteration of metabolic pathways}

We performed a metabolomic pathway enrichment analysis based on significantly altered metabolites $(\mathrm{p}<0.001)$, to identify pathways that contribute to the major differences when comparing samples taken before and after G-CSF administration (Fig. 4). This analysis identified altered glycogen metabolism as a major effect of G-CSF treatment, and metabolites belonging to pathways involved in nucleotide metabolism and amino sugar/acid metabolism were also over-represented in samples after G-CSF treatment.

\subsection{The metabolic alterations induced during G- CSF therapy are not caused by acetaminophen}

Musculoskeletal pain and flu-like symptoms are common during G-CSF therapy (Stroncek et al. 1996), and symptomatic relief can be achieved by acetaminophen (paracetamol). The detection of several metabolites of acetaminophen in the serum of stem cell donors indicates that paracetamol had been taken by some donors during G-CSF therapy. We therefore compared the metabolite 
Fig. 4 Metabolite pathway enrichment analysis to identify pathways enriched after G-CSF administration in healthy donors. A pathway enrichment analysis was done based on significantly altered metabolites with $\mathrm{p}<0.001$. Only signaling pathways with an enrichment value greater than two and at least two metabolites within each pathway are shown in the figure. The most significant p-values are seen in red, while the least significant are in yellow

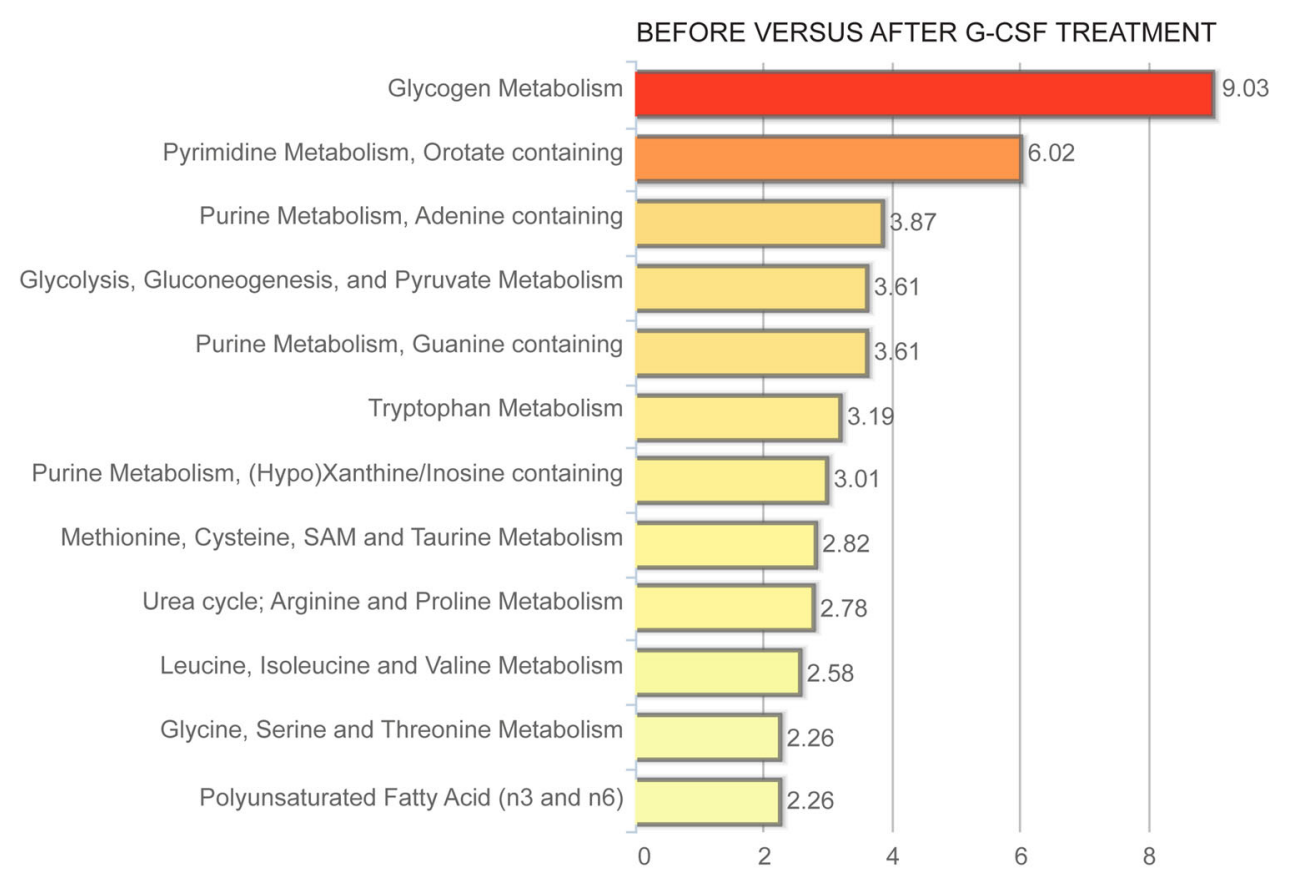

profile before and after G-CSF therapy for donors with high and low/undetectable levels of metabolites involved in acetaminophen metabolism. When comparing the levels of the 30 top-ranked metabolites (Fig. 2), we did not find any significant differences between the donor samples with high and low/undetectable paracetamol metabolite (data not shown). Thus, it seems unlikely that the paracetamol intake has a major impact on the metabolic modulation during G-CSF therapy.

\section{Discussion}

In this study, we investigated the early effects of G-CSF administration on the serum global metabolite profile of healthy stem cell donors that were younger than 65 years and had an adequate stem cell mobilization. G-CSF is mainly used as short-term therapy, generally requiring 4-6 days of treatment, for stem cell mobilization (Bendall and Bradstock 2014); however, it is also used as long-term treatment for patients with low-risk myelodysplastic syndrome (Jadersten et al. 2005) and especially for patients with chronic neutropenia (Dale 2016; Dale and Welte 2011; Donadieu et al. 2011; Zeidler et al. 2014). The suggested initial doses for congenital neutropenia are $3-5 \mu \mathrm{g} / \mathrm{kg}$ that are increased in steps of $5 \mu \mathrm{g} / \mathrm{kg}$ (Dale 2016; Donadieu et al. 2011), thus these G-CSF doses used in long-term therapy are also comparable to the doses used in our present study ( $5 \mu \mathrm{g} / \mathrm{kg}$ twice daily).

Our study included a relatively small number of samples, but our random forest classification analysis resulted in $97 \%$ predictive accuracy in differentiating the two groups, indicating that differences due to G-CSF administration were readily present. Several of the 30 top-ranking metabolites shown in Fig. 2 have been reported to be involved in biological processes such as regulation of immune responses, inflammation, vascular biology and epigenetic regulation (see Supplementary Table 3), though further studies will be needed to see if G-CSF has a longterm effect on these biological processes. In general, global metabolomics profiling revealed altered levels of lipids, amino acids, carbohydrates and nucleotides after administration with G-CSF.

One of the strongest changes in our dataset was the altered lipid metabolism, in particular the significantly higher levels of long-chain fatty acids as well as carnitineconjugated lipids after G-CSF administration, indicating changes in fatty acid $\beta$-oxidation. Long-chain fatty acids are conjugated to carnitine to facilitate transport across the mitochondrial membrane, and the increased acyl carnitine levels may thus suggest increased $\beta$-oxidation. Moreover, glycerol, a marker of lipolysis, was significantly decreased in the G-CSF treated group. These alterations may be due to increased fatty acid $\beta$-oxidation or alternatively due to disturbance of fatty acid oxidation resulting in increased amounts of lipid precursors. In addition, we found a decline in the serum levels of sphingosine 1-phosphate (S1P) after G-CSF administration, which is in concordance with another study that measured S1P levels in donors undergoing G-CSF-induced mobilization (Juarez et al. 2012). Disruption of fatty acid signaling has been implicated in mobilization of stem cells, in particular S1P (Ratajczak 
et al. 2010). To summarize, G-CSF treatment alters fatty acid metabolism and decreases the systemic levels of fatty acid metabolites involved in hydrolysis (phospholipid metabolism and lysolipids) whereas long/medium-chain fatty acids are generally increased.

In this study we found lower levels of branched chain amino acids and aromatic amino acids following G-CSF treatment. Among the aromatic amino acids we found altered levels of tryptophan and its degradation products which are shown to be associated with inflammation. Kynurenine plays a role in modulation of inflammation and the ratio of kynurenin/tryptophan has been suggested to be an indicator for the activity of indolamine-2,3-dioxygenase (IDO) (Widner et al. 1997), which can affect $\mathrm{T}$ cell functions. Branched chain amino acids can be substrates for both energy production and protein synthesis. They can be metabolized to give rise to intermediates for several metabolic pathways including the TCA cycle or fatty acid synthesis. Less amounts of the branched amino acids isoleucine, valine and leucine may thus potentially have an impact on energy metabolism by reduction of available metabolites. However, in addition to the lower levels of these amino acids, we also observed a general decrease in dipeptide levels after G-CSF administration which is supportive of reduced proteolysis.

Previous studies have examined effects of in vivo administration of G-CSF on normal peripheral blood mononuclear cells (PBMCs) using whole genome expression profiling. Changes in global gene expression profiles were then described both at early time points after G-CSF administration (up to 5 days) (Hernandez et al. 2005) and after 2-10 months (Amariglio et al. 2007), and even a year after G-CSF administration in $\mathrm{CD} 34^{+}$progenitor cells (Baez et al. 2014). These studies have revealed that there seems to be both early responses to G-CSF, transient changes that are normalized over time and more longlasting changes. In the recent study by Bàez et al., G-CSFmobilized hematopoietic progenitors had a difference in the expression of six microRNAs and even one year after G-CSF administration over 2424 genes maintained their altered expression (Baez et al. 2014). Among the differentially expressed genes were genes involved in cellular growth, cell death and survival, protein synthesis, gene expression and nucleic acid metabolism. In another study of twenty stem cell donors, changes of DNA methyltransferase activity in peripheral blood cells were found after G-CSF administration, though these changes returned to baseline within a week after apheresis (Leitner et al. 2014). Thus, several studies of donor cells suggest epigenetic changes induced after G-CSF administration in healthy donors, though none of these studies have investigated the global metabolite profile of healthy donors after G-CSF treatment. Accordingly, in our study of the systemic metabolite profile, we observed altered nucleic acid metabolism by G-CSF therapy, including lower levels of the purine nucleosides. Furthermore, several methylated products were increased which could suggest a difference in methylation potential through treatment with G-CSF. These results should be interpreted with great care, but could suggest that G-CSF has the potential to influence epigenetics.

We have not performed any functional assays to evaluate the potential association between metabolites altered by G-CSF and immunomodulatory effects; however, G-CSF has been shown to induce several cellular and immunological changes in donor cells (Anderlini et al. 1996; Shaw et al. 2015), and several of the altered metabolites found in our study have known immunoregulatory and/or angioregulatory effects, or can be markers of altered regulation of epigenetic/gene expression that may contribute to the previously described long-lasting effects after G-CSF therapy (Baez et al. 2014). Altered metabolite levels may reflect the direct effects of G-CSF on different immune cell types, affecting cell proliferation, differentiation and function, but also indirect effects through e.g. upregulation of cytokine production may subsequently affect cells and lead to altered metabolite levels. G-CSF binds to the single high-affinity $140 \mathrm{kDa}$ G-CSF receptor (G-CSFR), which is expressed on myeloid progenitor cells, mature granulocytes and monocytes, lymphocytes and endothelial cells (Demetri and Griffin 1991; Franzke et al. 2003) and can activate multiple signaling pathways including JAK-STAT and ERK/MAPK pathways. However, the expression of this receptor does not seem to be required for progenitor mobilization induced by G-CSF (Liu et al. 2000), and G-CSF-mediated effects may also occur independent of the G-CSF receptor. Further studies are needed to explore the mechanism inducing the metabolite changes after G-CSF treatment and their potential immunomodulatory effects and/or effects on other cell functions.

A possible explanation for our findings could be that they are secondary to an increased proliferation of immature hematopoietic cells to replace the cells that are lost from hematopoietic niches to the circulation. However, the CD $34^{+}$cell number and the total peripheral blood leukocyte number is controlled daily during stem cell mobilization, and the duration of this altered compartmentalization of hematopoietic cells is therefore relatively short $(<24 \mathrm{~h})$. Furthermore, the increased levels of immature hematopoietic cells in the peripheral blood probably represent a minor part of the overall number of nucleated bone marrow cells, and one should also emphasize that the cells have not yet been harvested at the time of sampling for metabolite analysis. However, we cannot exclude the possibility that the metabolic changes 
are secondary to an increased proliferation of bone marrow cells, but if so this is in our opinion most likely caused by direct G-CSF stimulated proliferation during the whole treatment period rather than being a compensatory mechanism to altered compartmentalization during the last hours before stem cell harvesting.

Several metabolites classified as xenobiotics were altered after G-CSF therapy. Many stem cell donors experience side effects during treatment (Stroncek et al. 1996), and acetaminophen is then recommended for pain relief. Some altered metabolites belonging to the xenobiotic class are a direct result of acetaminophen usage, and in our opinion some of the other metabolite changes may be associated with treatment toxicity and/or altered gastrointestinal function.

\section{Concluding remarks}

In totum, our results show that the level of several metabolites changed after G-CSF administration, primarily there were (i) increased levels of lipids indicating altered fatty acid metabolism, (ii) increased levels of methylated nucleosides, (iii) changes associated with energy metabolism, and (iv) altered levels of amino acids, including reduced peptide levels indicating decreased proteolysis and altered levels of acetylated peptides. Long-term follow up studies have concluded that the use of G-CSF to mobilize stem cells appears to be safe (Shaw et al. 2015). Our study shows distinct differences in the metabolite profiles between healthy donors before and after G-CSF administration; however this is only a snapshot of the metabolomic profile of donors at an early time point after G-CSF administration and further studies should examine the metabolite profiles over time, using a larger set of donors, to clarify whether G-CSF also has long-term effects on metabolite profiles and/or if patients on long-term G-CSF therapy should be monitored with regard to metabolic abnormalities.

Acknowledgements The authors would like to thank the Bergen Research Foundation (BFS), the Norwegian Cancer Society and Helse-Vest for financial support. We thank Edward Karoly from Metabolon, Inc., for helpful comments regarding data analysis of the metabolomics data.

\section{Compliance with ethical standards}

Conflicts of interest All authors declare that they have no conflicts of interest related to this manuscript.

Ethical approval All the authors declare that the procedures in this study were in accordance with the ethical standards of the responsible committee, approved by the local ethics committee in Bergen, Norway (REK Vest, 2011/996 and 2011/1237).
Informed consent Informed written consent was obtained from all individuals for being included in this study.

Open Access This article is distributed under the terms of the Creative Commons Attribution 4.0 International License (http://crea tivecommons.org/licenses/by/4.0/), which permits unrestricted use, distribution, and reproduction in any medium, provided you give appropriate credit to the original author(s) and the source, provide a link to the Creative Commons license, and indicate if changes were made.

\section{References}

Amariglio, N., et al. (2007). Changes in gene expression pattern following granulocyte colony-stimulating factor administration to normal stem cell sibling donors. Acta Haematologica, 117, 68-73.

Anderlini, P., Przepiorka, D., Champlin, R., \& Korbling, M. (1996). Biologic and clinical effects of granulocyte colony-stimulating factor in normal individuals. Blood, 88, 2819-2825.

Baez, A., et al. (2014). Granulocyte colony-stimulating factor produces long-term changes in gene and microRNA expression profiles in $\mathrm{CD}_{3}{ }^{+}$cells from healthy donors. Haematologica, 99 , 243-251.

Bendall, L. J., \& Bradstock, K. F. (2014). G-CSF: From granulopoietic stimulant to bone marrow stem cell mobilizing agent. Cytokine \& Growth Factor Reviews, 25, 355-367.

Buck, M. D., O'Sullivan, D., \& Pearce, E. L. (2015). T cell metabolism drives immunity. Journal of Experimental Medicine, $212,1345-1360$.

Dale, D. C. (2016). How I diagnose and treat neutropenia. Current Opinion in Hematology, 23, 1-4.

Dale, D. C., \& Welte, K. (2011). Cyclic and chronic neutropenia. Cancer Treatment and Research, 157, 97-108.

Demetri, G. D., \& Griffin, J. D. (1991). Granulocyte colonystimulating factor and its receptor. Blood, 78, 2791-2808.

Donadieu, J., Fenneteau, O., Beaupain, B., Mahlaoui, N., \& Chantelot, C. B. (2011). Congenital neutropenia: Diagnosis, molecular bases and patient management. Orphanet Journal of Rare Disease, 6, 26.

Evans, A. M., et al. (2014). High resolution mass spectrometry improves data quantity and quality as compared to unit mass resolution mass spectrometry in high-throughput profiling metabolomics. Metabolomics, 4, 132.

Franzke, A., et al. (2003). G-CSF as immune regulator in T cells expressing the G-CSF receptor: Implications for transplantation and autoimmune diseases. Blood, 102, 734-739.

Hernandez, J. M., et al. (2005). Mobilisation with G-CSF in healthy donors promotes a high but temporal deregulation of genes. Leukemia, 19, 1088-1091.

Jadersten, M., Montgomery, S. M., Dybedal, I., Porwit-MacDonald, A., \& Hellstrom-Lindberg, E. (2005). Long-term outcome of treatment of anemia in MDS with erythropoietin and G-CSF. Blood, 106, 803-811.

Juarez, J. G., et al. (2012). Sphingosine-1-phosphate facilitates trafficking of hematopoietic stem cells and their mobilization by CXCR4 antagonists in mice. Blood, 119, 707-716.

Leitner, G. C., Faschingbauer, M., Wenda, S., Weigel, G., \& Fischer, G. (2014). Administration of recombinant human granulocytecolony-stimulating factor does not induce long-lasting detectable epigenetic alterations in healthy donors. Transfusion, $54,3121-3126$. 
Liu, F., Poursine-Laurent, J., \& Link, D. C. (2000). Expression of the G-CSF receptor on hematopoietic progenitor cells is not required for their mobilization by G-CSF. Blood, 95, 3025-3031.

Malard, F., et al. (2016). Larger number of invariant natural killer $\mathrm{T}$ cells in PBSC allografts is associated with improved GVHDfree, progression-free survival. Blood, 127, 1828-1835.

Pabst, C., Schirutschke, H., Ehninger, G., Bornhauser, M., \& Platzbecker, U. (2007). The graft content of donor T cells expressing gamma delta $\mathrm{TCR}+$ and $\mathrm{CD} 4+$ foxp $3+$ predicts the risk of acute graft versus host disease after transplantation of allogeneic peripheral blood stem cells from unrelated donors. Clinical Cancer Research, 13, 2916-2922.

Panopoulos, A. D., \& Watowich, S. S. (2008). Granulocyte colonystimulating factor: Molecular mechanisms of action during steady state and 'emergency' hematopoiesis. Cytokine, 42, 277-288.

Ratajczak, M. Z., et al. (2010). Novel insight into stem cell mobilization-plasma sphingosine-1-phosphate is a major chemoattractant that directs the egress of hematopoietic stem progenitor cells from the bone marrow and its level in peripheral blood increases during mobilization due to activation of complement cascade/membrane attack complex. Leukemia, 24, 976-985.

Rezvani, K., et al. (2006). High donor FOXP3-positive regulatory T-cell (Treg) content is associated with a low risk of GVHD following HLA-matched allogeneic SCT. Blood, 108, 1291-1297.

Rutella, S., Zavala, F., Danese, S., Kared, H., \& Leone, G. (2005). Granulocyte colony-stimulating factor: A novel mediator of $\mathrm{T}$ cell tolerance. Journal of Immunology, 175, 7085-7091.

Shaw, B. E., Confer, D. L., Hwang, W., \& Pulsipher, M. A. (2015). A review of the genetic and long-term effects of G-CSF injections in healthy donors: A reassuring lack of evidence for the development of haematological malignancies. Bone Marrow Transplantation, 50, 334-340.

Stroncek, D. F., et al. (1996). Treatment of normal individuals with granulocyte-colony-stimulating factor: Donor experiences and the effects on peripheral blood $\mathrm{CD} 34^{+}$cell counts and on the collection of peripheral blood stem cells. Transfusion, 36, 601-610.

Widner, B., Werner, E. R., Schennach, H., Wachter, H., \& Fuchs, D. (1997). Simultaneous measurement of serum tryptophan and kynurenine by HPLC. Clinical Chemistry, 43, 2424-2426.

Zeidler, C., et al. (2014). Outcome and management of pregnancies in severe chronic neutropenia patients by the European Branch of the Severe Chronic Neutropenia International Registry. Haematologica, 99, 1395-1402. 